\title{
PERFIL DE LA SITUACIÓN DE LA MUJER EN LAS COOPERATIVAS DE TRABAJO EN ESPAÑA
}

\author{
POR \\ Paloma ARROYO SÁNCHEZ ${ }^{1}$
}

\section{RESUMEN:}

El artículo analiza los datos de un estudio sobre la situación de la mujer en las cooperativas de trabajo: su vinculación con la cooperativa, categoría profesional, cualificación, conciliación laboral y familiar, en comparación con una investigación realizada en 2004, así como respecto de la mujer en España y UE.

Palabras clave: Mujer, cooperativa de trabajo, empleo femenino, formación, conciliación: personal y laboral.

Claves ECONLIT: B540, J210, J540

\section{PROFILE OF THE SITUATION OF WOMEN IN WORKER'S COOPERATIVES IN SPAIN}

\begin{abstract}
:
The article analyzes data from a study on the situation of women in the cooperative work: its relationship with the cooperative, professional, qualifications, work-life balance, compared to a survey conducted in 2004 and on the Women in Spain and EU.

\footnotetext{
${ }^{1}$ Directora de la Confederación Española de Cooperativas de Trabajo Asociado COCETA, calle Vallehermoso, 15 Madrid 28015 (España). Direcciones de correo electrónico: confederacion@coceta.coop y paloma@coceta.coop
}

REVESCO Nº 105 - MONOGRÁFICO: La economía social y la igualdad de género -

ISSN: 1885-8031 - www.ucm.es/info/revesco

DOI: 10.5209/rev_REVE.2011.v105.5

Fecha de recepción: 23/11/2010

Fecha de aceptación: 01/03/2011 
Keywords: Woman, cooperative work, feminine employment, formation, conciliation: personal and labor.

\section{INTRODUCCIÓN}

Hasta el año 2004, los estudios y documentos referidos a la situación de la Mujer en España, en un ámbito tan específico como las cooperativas de trabajo, prácticamente no existían.

Ante esta situación, en el año citado, se procedió a realizar una investigación que permitiera conocer el perfil personal, familiar y laboral de las mujeres que trabajaban en las cooperativas de trabajo. La investigación se dirigió a cooperativas de trabajo, pertenecientes a diversos sectores de actividad económica, con dimensiones empresariales diferentes y de la totalidad del Estado Español.

El resultado de la investigación se recogió en el estudio denominado: La realidad social y laboral de las mujeres en el Cooperativismo de trabajo asociado en España. ${ }^{2}$

Transcurridos cinco años, se ha considerado necesario realizar la actualización del citado Estudio. Pues, si bien es cierto, que durante este período de tiempo, las estadísticas de algunas instituciones y entidades - públicas y privadas- se han perfeccionado, como por ejemplo las de la Dirección General de la Economía Social o del Instituto de la Mujer, no lo es menos, que la situación de la mujer en la cooperativa de trabajo permanece, en una buena parte de sus diversas facetas, desconocida e invisible. Desconocimiento que alcanza no sólo a la dimensión estadística, al campo de las investigaciones, de los estudios o de los informes, sino que abarca, también, a la sociedad, en general.

Conocer el perfil de la situación de la mujer en la cooperativa de trabajo, permitirá, en función de los resultados obtenidos: desarrollar herramientas formativas para mejorar su

\footnotetext{
${ }^{2}$ El estudio sobre La realidad social y laboral de las mujeres en el Cooperativismo de trabajo asociado en España puede ser consultado en www.coceta.coop.
}

REVESCO No 105 - MONOGRÁFICO: La economía social y la igualdad de género -

ISSN: 1885-8031 - www.ucm.es/info/revesco 
desarrollo profesional, implementar medidas que permitan hacer de la cooperativa de trabajo un modelo de gestión empresarial adecuado; difundir la idoneidad de la cooperativa, como referente empresarial para las mujeres.

Si a todo ello añadimos que las cooperativas de trabajo son empresas singulares, con unos principios y valores que hacen de la persona, el eje fundamental; que se crean para proporcionar puestos de trabajo, estables y de calidad, para las personas que las forman; que es reconocido su prestigio como entidades generadoras de empleo, tanto en periodos de crisis económica, como en épocas de bonanza; que un amplio volumen de empleos pertenece a mujeres, justifica y motiva el Estudio, del que se ofrece una síntesis en este artículo.

\section{DESCRIPCIÓN DE LA SITUACIÓN ACTUAL DE LA MUJER EN ESPAÑA Y EN LA UE, EN FUNCIÓN DE DIVERSAS VARIABLES}

\subsection{El Empleo en perspectiva de género.}

Según los datos del INE a 01/01/2010 la población en España era de 46.951.532 personas, de las cuales 23.756 .098 son mujeres, un 50,6\%, prácticamente algo más de la mitad de la población. De ellas, 10.139.300 son mujeres activas, es decir, aquellas que están en edad de poder trabajar, si bien de dicho número 1.934 .000 se encuentran en paro. ${ }^{3}$

La tasa de empleo - considerada una de las variables más relevante desde el punto de vista económico, ya que afecta a todo el sistema productivo- se situaba en las mujeres, al finalizar el cuarto trimestre de 2009 , en el $41,8 \%$ en tanto que en los hombres lo hacía en el $55,4 \%$. Esta notable diferencia entre géneros muestra la necesidad de crear y difundir políticas activas generadoras de empleo para las mujeres.

A lo largo del último año y medio, en el que la crisis económica y financiera se ha disparado y no sólo en España o en la Unión Europea y su zona de influencia, sino en todo el mundo, hay que destacar que en España, los niveles de empleo en la mujer, se han

\footnotetext{
${ }^{3}$ A fecha 01-01-2010. Datos Ministerio de Trabajo e Inmigración.www.mtin.es
}

REVESCO No 105 - MONOGRÁFICO: La economía social y la igualdad de género -

ISSN: $1885-8031$ - www.ucm.es/info/revesco 
mantenido. Niveles de empleo ciertamente sostenidos, del 43,9\% de finales de 2007, al $43,6 \%$ al final del 2008, y del 41,8\% a finales del 2009, tal como se ha indicado, con una disminución, a pesar de la crisis económica, de sólo un 2,1\%.

Si comparamos el nivel de empleo en la población masculina, para el mismo periodo, se observa que, también, se ha producido una minoración aunque mayor que en el caso de las mujeres, alcanzando el $9,1 \%$.

Este menor descenso de la población femenina empleada, respecto de la masculina, en un período de crisis económica, responde a varios factores, unos de carácter sectorial, al haber sido el sector de la construcción y los subsectores dependientes, los que han generado un mayor volumen de empleos destruidos, radicados fundamentalmente, en los hombres, dada la peculiaridad del citado sector; otro de los factores, como ya ha sido puesto de manifiesto por algunos estudios ${ }^{4}$, es el referido a que un mayor número de mujeres activas "ha salido a buscar trabajo", así en el 2009, 100.000 mujeres-amas de casa- salieron en búsqueda de un empleo, al tiempo que 42.000 hombres comenzaron a realizar las tareas del hogar.

En España, la presencia de la mujer en el mundo laboral dista de alcanzar los niveles europeos; si comparamos la tasa de empleo del 41,8\% con la media de empleo femenino de la Unión Europea (UE-27) a finales de 2008, comprobaremos que se sitúa en más de dieciocho puntos por debajo, ya que en la UE es del $59,1 \%{ }^{5}$. Concretando, aún más, once países situaban su tasa de empleo, entre el $60 \%$ y el $70 \%$, colocándose a la cabeza de todos ellos Dinamarca, donde la tasa de empleo de las mujeres llega al $74,3 \%$, seguida por Suecia $(71,8 \%)$ y Países Bajos $(71,1 \%)$.

España, además de no alcanzar estas tasas de empleo femeninas, está por debajo de diecinueve países de la UE.

Actualmente existe el gran problema de crear empleos, tanto masculinos como femeninos, problema que no sólo afecta a España, aun cuando sea donde esté resultando más

\footnotetext{
${ }^{4}$ Informe Fundación Adecco. IV Informe Perfil de la Mujer Trabajadora. 01 Marzo 2010

${ }^{5}$ Eurostat. 18 de octubre de 2009
}

REVESCO N 105 - MONOGRÁFICO: La economía social y la igualdad de género -

ISSN: 1885-8031 - www.ucm.es/info/revesco 
complicado encontrar vías de solución, sino a la práctica totalidad de países de la UE, que han comprobado como los objetivos de la Estrategia de Lisboa, que estimaban una cifra de empleo del $70 \%$ en el 2010, son inalcanzables; por ello, se ha comenzado a trabajar, en la que se ha dado en denominar, Estrategia Europa 2020. Su finalidad es el alcanzar un crecimiento inteligente, sostenible e integrador, fomentando una economía que permita un alto nivel de empleo que tenga cohesión social y territorial ${ }^{6}$.

\subsection{La Retribución como variable para fomentar la equidad de género.}

Desde la perspectiva de género, ¿Cómo se comporta la variable del salario o la remuneración, en España? ¿Y en la UE? Es de reseñar que las mujeres en la UE ganan de media un 17,4\% menos que los hombres y, en España, este porcentaje se eleva en unas décimas, alcanzando prácticamente el $18 \%$, sin que parezca que en el contexto actual, pueda mejorar esta situación.

Por países, las mayores diferencias se producen en Estonia (30\%) y Austria (25\%), en tanto que en Reino Unido, los hombres ganan hasta un $22 \%$ más que las mujeres y en Alemania, sube hasta el 23\%. Las cifras más bajas se corresponden como Portugal (9\%), Italia (6\%) y Malta (5\%).

Los cálculos de la Comisión Europea indican que un 50\% de las desigualdades salariales en la Unión Europea se deben a una discriminación directa, es decir, a una remuneración inferior por el mismo trabajo y, la otra mitad, a la dificultad de acceso para las mujeres a buenos trabajos, a pesar de que ellas representan el 59\% de los nuevos licenciados universitarios.

De esta forma, mientras que el nivel de empleo de las mujeres está aumentando, de forma constante, en los últimos años (un 58,3\% de las mujeres frente un 72,5\% de los hombres), las mujeres aún trabajan por horas (trabajo parcial) con más frecuencia que los hombres (un $31,2 \%$ de las mujeres, frente a un 7,7\% de los hombres), predominando en los

\footnotetext{
${ }^{6}$ Comunicación de la Comisión. Europa 2020. Una estrategia para un crecimiento inteligente, sostenible e integrador. Bruselas, 3.3.2010. COM(2010) 2020
}

REVESCO No 105 - MONOGRÁFICO: La economía social y la igualdad de género -

ISSN: 1885-8031 - www.ucm.es/info/revesco 
sectores con salarios más bajos (más del $40 \%$ de las mujeres trabajan en la sanidad, la educación o la administración pública; prácticamente el doble que los hombres).

Es preciso reseñar que la diferencia de retribución también reduce los ingresos y las pensiones de las mujeres durante toda la vida, lo que al final genera pobreza: un $21 \%$ de las mujeres de más de 65 años corren riesgo de pobreza, frente a un 16\% de los hombres, al tiempo que, este menor ingreso de las mujeres, puede afectar al propio Estado del Bienestar. En España, esto se produce, debido a la existencia de un sistema basado en la recaudación fiscal procedente del trabajo, de tal manera que la menor remuneración de las mujeres, implica un menor ingreso para el estado; lo que, a su vez, genera una carencia de suficientes recursos para sustentar el Estado del Bienestar, y produce cuantías inferiores en las pensiones. Se configura, de esta forma, un círculo, del que se ha de salir mediante el correspondiente reconocimiento del nivel salarial o retributivo de las mujeres.

Esta variable de la desigual retribución entre mujeres y hombres parece ser una constante en todos los países de la UE, no obstante las diferencias existentes entre unos países y otros.

\subsection{La Formación como variable de acceso al mundo laboral}

El grado/ nivel de formación que poseen las mujeres que trabajan en España y en la UE se convierte en otra de las variables a analizar. Cabe señalar que siguiendo los informes de la $\mathrm{OCDE}^{7}$, se constata que el nivel de formación de las personas adultas, en España, se encuentra por debajo de la media de los demás países europeos.

Así, en España, el nivel educativo de la población adulta (de 25-a 64 años), se mantiene por debajo de la media de los países de la OCDE y de Europa. El dato más relevante es que el $50 \%$ de la población en España ha completado, como máximo, la Educación Secundaria Obligatoria (ESO), un porcentaje muy superior a la media de la OCDE y de la

\footnotetext{
${ }^{7}$ Education at a Glance 2008. Informe de la Organización para la Cooperación y el Desarrollo Económico (OCDE), Panorama de la educación 2008. Ministerio de Educación y Ciencia.
}

REVESCO No 105 - MONOGRÁFICO: La economía social y la igualdad de género -

ISSN: $1885-8031$ - www.ucm.es/info/revesco 
Unión Europea, en donde dicho porcentaje, para este nivel de estudios desciende al $31 \%$. Sólo Portugal con el 72\% supera el porcentaje español.

Algo similar ocurre en el Bachillerato o en la Formación Profesional de Grado Medio. La media europea, que ha completado estos estudios, se sitúa en el $45 \%$, en tanto que en España, únicamente, un $21 \%$ de la población ha superado este nivel educativo.

En lo referente a la Educación Superior, España aparece con datos positivos. La población española que completa estudios universitarios o de FP de Grado Superior (28\%) es sensiblemente superior a la media de la OCDE (27\%) y de la Unión Europea (24\%). Ello, es debido, en gran parte, al elevado porcentaje de población que en otros países accede a la Formación Profesional de Grado Medio, como vía de incorporación al mercado laboral. Su fundamento radica en que el sistema de acceso a la vida laboral, se encuentra regulado de tal forma que, la obtención de títulos de formación profesional, constituyen nivel suficiente para acceder a un puesto de trabajo, lo que hace poco necesario un título de nivel superior. En la práctica existe una mayor coordinación entre los estudios profesionales y los empleos ofertados.

Es destacable asimismo, la importante diferencia entre los hombres y mujeres que han finalizado sus estudios en la Educación Superior, así la tasa de hombres que se gradúan en una universidad es del 25,5\%, y la de mujeres se eleva al 40,8\%. Igualmente sucede en la Formación Profesional de Grado Superior, donde las mujeres graduadas (16\%) superan a los hombres $(13 \%)$.

Otra de las variables que la OCDE analiza dentro de su informe "Education at a Glance 2008" es la relación vigente entre la tasa de empleo de los países estudiados y el nivel de formación de su población. A este respecto una de las conclusiones que indica el Informe es que tanto en España como en el conjunto de los países de la OCDE un mayor nivel de formación significa un porcentaje más bajo de desempleo y un sueldo más elevado.

En el caso concreto de España, las diferencias son sustanciales. Entre la población masculina de 25 a 64 años hay una diferencia de hasta 20 puntos, en función del nivel 
educativo alcanzado. De este modo, la tasa de empleo de los hombres que tienen, únicamente, estudios primarios es del 69\%, mientras que la de los hombres que cuentan con un título universitario se eleva al $88 \%$. Este indicador demuestra aún más este principio en la población femenina. La tasa de empleo de mujeres con estudios primarios es de un $32 \%$ y, sin embargo, la de las mujeres que han alcanzado una formación universitaria se eleva al $80 \%$.

Esta correlación (formación - empleo) también se manifiesta, respecto de las mujeres cuando se trata de su participación en la que se denomina Formación Continua, conceptuada como toda actividad de aprendizaje, que se realiza a lo largo de la vida, con el objetivo de mejorar las competencias, los conocimientos y las aptitudes relacionados con el empleo. Así según los datos, ofrecidos por la Fundación Tripartita para la Formación y el Empleo, correspondientes al Informe de Resultados del año 2006, el porcentaje de mujeres formadas se eleva al 45,2\%; porcentaje superior al que representan respecto de la población ocupada, que se situaba en el 38,7\%; el porcentaje de mujeres formadas se eleva en siete puntos respecto del año 2005. Este porcentaje de mujeres formadas se produce tanto si se trata de la denominada Formación de Oferta o de la denominada de Demanda. De los datos de la Fundación Tripartita para la Formación y el Empleo, se deduce que el porcentaje de presencia de la mujer en la actividad formativa supera su peso respecto del porcentaje de población ocupada. Así en el 2006 se incrementa la participación en la formación en tanto que se reduce en el mismo porcentaje de incremento en el empleo, de forma tal que se presume que una buena formación en el puesto de trabajo o bien en otra profesión o actividad, capacitará para mantener el empleo, o incluso encontrarlo. Los datos de los años 2005 y 2006 muestran este principio, de tal manera que se forman más mujeres cuando es menor su tasa de empleo y viceversa, tal y como se acredita con los Gráficos siguientes.

REVESCO No 105 - MONOGRÁFICO: La economía social y la igualdad de género -

ISSN: 1885-8031 - www.ucm.es/info/revesco 
Gráfico 1: Correlación del porcentaje de mujeres formadas y ocupadas en el año 2005

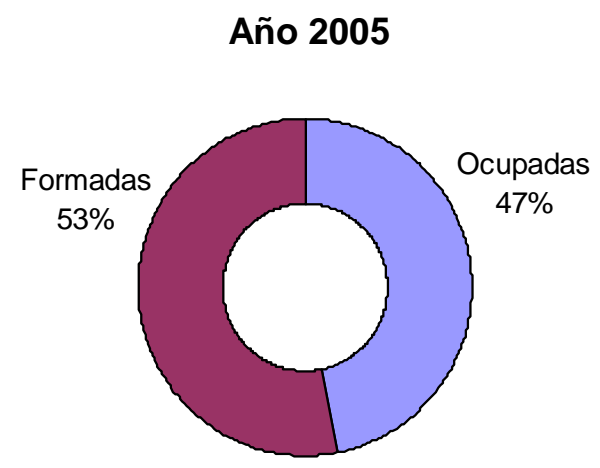

Fuente: Fundación Tripartita para la Formación y el Empleo

Gráfico 2: Correlación del porcentaje de mujeres formadas y ocupadas en el año 2006

Año 2006

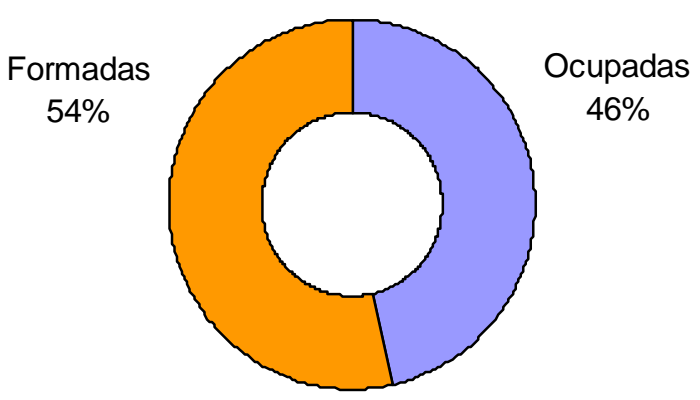

Fuente: Fundación Tripartita para la Formación y el Empleo

\subsection{Conciliación de la vida laboral y familiar}

La última de las variables que se consideró en la investigación fue la relativa a la conciliación de la vida laboral y familiar.

La Ley 39/1999, de 5 de noviembre, para promover la conciliación de la vida familiar y laboral de las personas trabajadoras, indica en su Exposición de Motivos

"La necesidad de conciliación del trabajo y la familia ha sido ya planteada a nivel internacional y comunitario como una condición vinculada de forma inequívoca a la nueva realidad social. Ello plantea una compleja y difícil problemática que debe

REVESCO No 105 - MONOGRÁFICO: La economía social y la igualdad de género -

ISSN: 1885-8031 - www.ucm.es/info/revesco 
abordarse, no sólo con importantes reformas legislativas, como la presente, sino con la necesidad de promover adicionalmente servicios de atención a las personas, en un marco más amplio de política de familia.

En este sentido, en la IV Conferencia mundial sobre las mujeres, celebrada en Pekín en septiembre de 1995, se consideró como objetivo estratégico fomentar una armonización de responsabilidades laborales y familiares entre hombres y mujeres y en la Declaración aprobada por los 189 Estados allí reunidos, se reafirmó este compromiso.

Por su parte, en el ámbito comunitario, la maternidad y la paternidad, en su más amplio sentido, se han recogido en las Directivas del Consejo 92/85/CEE, de 19 de octubre, y 96/34/CE, del Consejo, de 3 de junio. La primera de ellas contempla la maternidad desde el punto de vista de la salud y seguridad en el trabajo de la trabajadora embarazada, que haya dado a luz o en periodo de lactancia. La segunda, relativa al Acuerdo marco sobre el permiso parental, celebrado por la UNICE, el CEEP y la CES, prevé el permiso parental y la ausencia del trabajo por motivos de fuerza mayor como medio importante para conciliar la vida profesional y familiar y promover la igualdad de oportunidades y de trato entre hombres y mujeres.

Mediante la presente Ley se completa la transposición a la legislación española de las directrices marcadas por la normativa internacional y comunitaria superando los niveles mínimos de protección previstos en las mismas”.

A través de la citada norma se pretendía llevar a la base de la negociación colectiva los convenios colectivos- la necesidad de facilitar que las mujeres y los hombres pudieran compaginar su vida laboral y familiar. A partir del año 2000 se fueron introduciendo en los diferentes convenios colectivos las cláusulas necesarias, para ir avanzando en esta dirección. Al mismo tiempo, además de esta actuación, se inició una campaña para difundir los conceptos de la ley en el mundo del trabajo y, aún más importante, en la sociedad. Así en el primer estudio realizado por Confederación Española de Cooperativas de Trabajo Asociado (COCETA) ya se contemplaba esta variable de la conciliación en las cooperativas de trabajo y, ya en aquél momento, los resultados de la investigación dejaron patente cómo en las 
cooperativas de trabajo la conciliación de la vida laboral y familiar era una realidad, más allá de la propia existencia de la ley. Primaba la estructura democrática de la cooperativa, como base para promover la mejora de las condiciones de vida de las personas que la conforman; por ello, en el siguiente estudio de Confederación Española de Cooperativas de Trabajo Asociado (COCETA) resultó de gran importancia conocer cómo había evolucionado o involucionado esta variable, ya que en el resto de la sociedad, más allá de lo establecido en los textos de los diferentes convenios colectivos, los logros en la mejora de la conciliación laboral y familiar de las personas trabajadoras, habían resultado más bien escasos.

Es preciso recordar que la mayor dedicación de las mujeres a las tareas de los cuidados familiares, constituye el elemento central para el mantenimiento de los niveles de su discriminación laboral, así como su acceso al empleo, a la formación, a la promoción y a un reconocimiento profesional, lo que produce consecuencias en el reconocimiento retributivo; por ello, se ha de lograr que la mujer participe en actividades formativas y se integre laboralmente, sin que ello signifique una carga adicional a las tareas del cuidado del hogar y la familia, objetivo que como ya se ha señalado, no llegó a cumplir la Ley 39/1999, de conciliación de la vida familiar y laboral de las personas trabajadoras, tal y como se desprende cuando se analizan los denominados "usos del tiempo"

“.. los avances en cuanto al tiempo dedicado por el hombre al trabajo doméstico, en los últimos diez años, apenas son perceptibles, y, sin embargo en el caso de la mujer, su evolución en el incremento del tiempo dedicado al trabajo es constatable, disminuyendo el tiempo dedicado al hogar, así como el relativo al tiempo de ocio".

La falta de obtención de resultados positivos de la Ley 39/1999, llevó al gobierno a la promulgación de una nueva ley, la 3/2007, Ley Orgánica para la Igualdad Efectiva de Mujeres y Hombres, que se planteó con un alcance y objetivos más ambiciosos. Para alcanzarlos, el gobierno realizó un Plan estratégico de igualdad de oportunidades 2008$2011^{8}$. En el ínterin de los resultados que pueda ir ofreciendo el Plan Estratégico, se están

\footnotetext{
${ }^{8}$ La brecha existente entre la igualdad legal y la igualdad real hace necesario que los poderes públicos pongan en marcha políticas públicas orientadas a superar la discriminación y garantizar la igualdad. La Ley Orgánica para la Igualdad Efectiva de Mujeres y Hombres establece el fundamento jurídico para avanzar hacia la efectiva igualdad en todos los ámbitos de la vida social, económica, cultural y política. Su aplicación requiere de un Plan Estratégico que concrete los objetivos, ámbitos y medidas de actuación en los que los poderes públicos han de centrar sus acciones (Art. 17 de la LOIEMH). La coordinación entre los distintos niveles de las Administraciones
}

REVESCO No 105 - MONOGRÁFICO: La economía social y la igualdad de género -

ISSN: 1885-8031 - www.ucm.es/info/revesco 
publicando algunos estudios de evaluación, si bien en campos concretos, como la Evaluación de políticas de igualdad en el ámbito laboral en España (año 2007-2008) ${ }^{9}$.

En cualquier caso, se ha de ser realista, en el sentido de que las previsiones que se realizaron para el Plan Estratégico se han visto alteradas, ante la irrupción en el panorama socio-económico de la crisis económica y financiera, que ha generado situaciones no previstas, por lo que, tal vez sería conveniente y necesaria una revisión en función de las circunstancias existentes en la actualidad.

Hasta aquí, se ha recogido la situación legislativa existente en España, en relación a la conciliación de la vida laboral y familiar de la mujer, procediendo ahora a realizar alguna aproximación, aunque cuando fuere menor, al ámbito de la UE.

En dicho ámbito, encontramos el estudio Reconciliation between work, private and family life in the European Union ${ }^{10}$, en el que se recogen las principales magnitudes al respecto, y, que de forma concreta en lo referido al uso del tiempo nos ofrece la gráfica siguiente $^{11}$,

Públicas (general, autonómica y local) y los distintos estamentos sociales hace necesario, asimismo, la elaboración de dicho Plan Estratégico.

El Plan Estratégico (2009-2011) desarrolla cuatro principios rectores: Ciudadanía, Empoderamiento, Transversalidad e Innovación, que están, además, relacionados entre sí.

1. La redefinición del modelo de ciudadanía en concordancia con la igualdad de género, que entiende la igualdad más allá de la equiparación de lo femenino con lo masculino y considera lo femenino como riqueza; que afirma la libertad femenina y atiende a la singularidad y pluralidad de las mujeres, de modo que no se las contemple como "lo colectivo femenino".

2. El empoderamiento de las mujeres, en el sentido de valorar y fortalecer sus formas de hacer, de ejercer el poder y de relacionarse. El concepto de empoderamiento posee una doble vertiente. Por una parte, se refiere a la capacidad de las mujeres para acceder a aquellos puestos donde se toman decisiones. Por otra, a la revalorización de la aportación de las mujeres.

3. La transversalidad de la perspectiva de género, como herramienta que busca modificar las formas actuales de la política, de modo que se tomen como referencia las experiencias, las aportaciones de las mujeres, su modo de estar en el mundo y su conocimiento.

4. La innovación científica y tecnológica, como una de las principales fuerzas de cambio social. Aunque su dominio confiere un enorme poder, ya que quien controla la tecnología controla el futuro, las mujeres han estado excluidas de dichos ámbitos por medio de barreras formales e informales. (www.migualdad.es).

9 Evaluación de políticas de igualdad en el ámbito laboral en España (año 2007-2008). www.inmujer.migualdad.es/MUJER/mujeres/estud_inves/828.pdf

${ }^{10}$ Reconciliation between work, private and family life in the European Union. Eurostat. Statistical books.2009 Edition

${ }^{11}$ Reconciliation between work, private and family life in the European Union. Eurostat. Statistical books.2009 Edition. Page. 43

REVESCO No 105 - MONOGRÁFICO: La economía social y la igualdad de género -

ISSN: 1885-8031 - www.ucm.es/info/revesco 
Gráfico 3: Indicadores de tiempo de trabajo, por grupos de países y género, 2005

Flgure 3,3; Composite indicator of working time, by country group and gender, 2005 (hours)

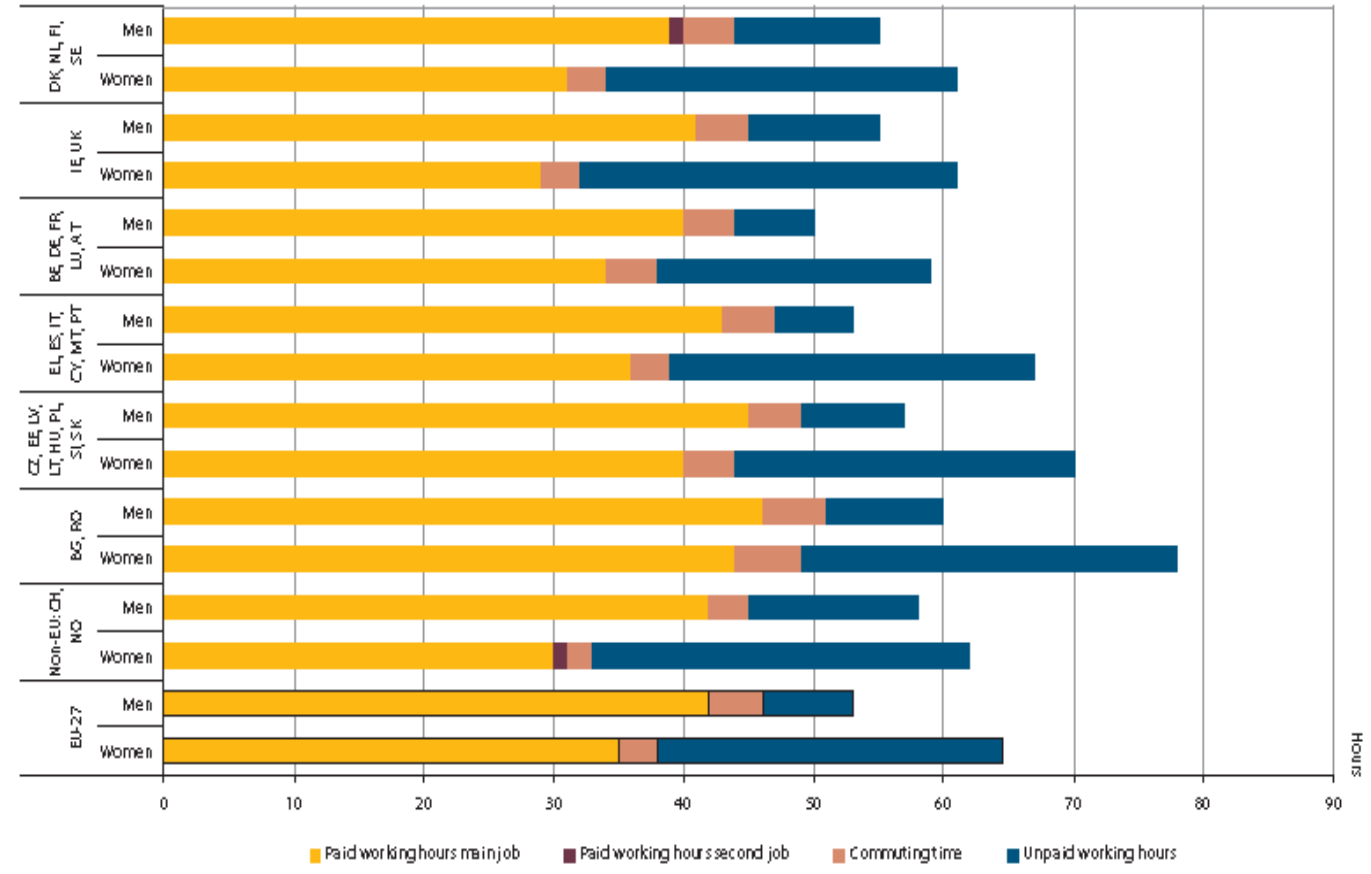

Motei The oom posite working hours indicator is made up of the wexkly working hours, plus the average weekly working hours in jobs other than the main job, commuting time and the total weekly unpaid working hours, declared by male and fern ale workers aged 15 or over,

Soure: EWS.

En la misma se aprecia que en todos los países de la UE (27) en el 2005, el tiempo que los hombres empleaban en los trabajos no remunerados - trabajos domésticos- es notablemente inferior al de las mujeres. En el Gráfico 3, aparece la primera parte de la barra que se corresponde con el trabajo remunerado, la segunda, más corta, con otros trabajos remunerados- segundo trabajo-, apareciendo, también, los trabajos a la comunidad, siendo la última parte de la barra la que representa el trabajo no remunerado.

Bien es cierto que los datos están referenciados al 2005, y, en este sentido, desde entonces, se han incentivado desde la UE políticas para favorecer la igualdad entre mujeres y hombres, así como para conciliar la vida laboral y familiar. La puesta en marcha de estas políticas aún no ha ofrecido resultados visibles y, como en el caso de España, la existencia de la crisis económica, es probable que pueda dificultar el logro de este objetivo.

Como resumen y para una mejor visualización de esta gráfica se ofrece, ampliada, la parte correspondiente a la media de la Unión Europea, en la que se constata lo señalado en el párrafo anterior.

REVESCO No 105 - MONOGRÁFICO: La economía social y la igualdad de género -

ISSN: 1885-8031 - www.ucm.es/info/revesco 
Gráfico 4: Indicador de la media de la UE, de horas de trabajo y género

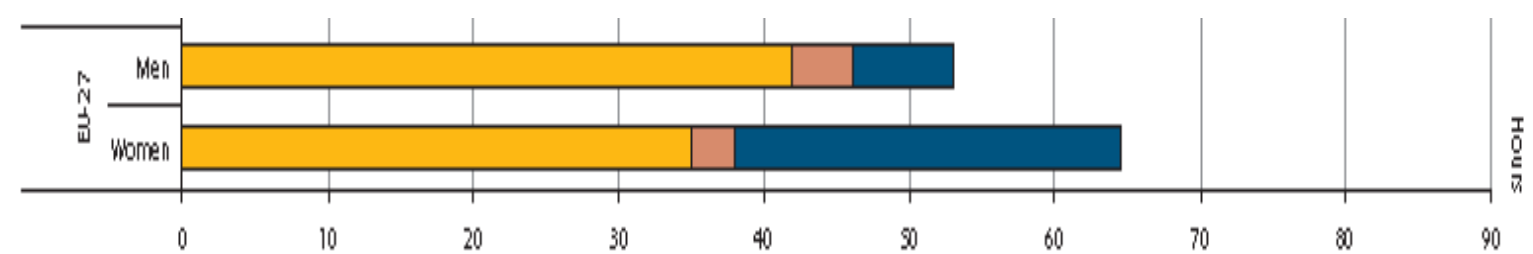

El gráfico muestra en la primera parte de las barras las horas dedicadas al trabajo remunerado, la segunda las horas de desplazamientos y la última parte de la barra, las dedicadas al trabajo no remunerado - trabajo doméstico-. En la citada ampliación del gráfico aparece de forma clara la mayor realización de horas de trabajo no pagadas (25,5 horas) por las mujeres, en relación a las horas dedicadas por los hombres; en tanto que los hombres dedican más horas $(41,7)$ al trabajo remunerado. Todo ello se ha realizado con una base de un total de 90 horas. A través de estas gráficas, se puede comprobar, la existencia de una menor conciliación de la vida familiar y laboral de la mujer en el ámbito de la UE.

\section{PERFIL DE LAS COOPERATIVAS DE TRABAJO Y LA MUJER EN ESPAÑA}

Las cooperativas, se consideran instrumentos útiles para acometer políticas de desarrollo económico y bienestar social, debido a varios factores (Vara, 1994, p. $\left.13^{12}\right)$ :

1. Son empresas muy flexibles en cuanto al tamaño y a la movilidad de sus socios.

2. Contribuyen a la formación de un espíritu empresarial.

3. Permiten ajustes bastante rápidos del salario real, tanto a la baja como al alza, según la coyuntura económica, y, por tanto, producen efectos favorables en el sostenimiento del empleo.

4. Por propia exigencia de los principios cooperativos, deben invertir en la formación de su capital humano y tomar las decisiones de forma participativa.

\footnotetext{
${ }^{12}$ Vara, M.J. (1994). Funciones de la economía social en el modelo de desarrollo económico. I.C.E., nº 729, pp. 9-17.
}

REVESCO No 105 - MONOGRÁFICO: La economía social y la igualdad de género -

ISSN: 1885-8031 - www.ucm.es/info/revesco 
En consecuencia, contribuyen a elevar el nivel de cualificación global del factor trabajo vinculando formación-experiencia-compromiso.

Así, decir cooperativa de trabajo, es decir:

- Empresa

- Autoempleo colectivo

- Primacía de la persona, por encima del capital

- Democracia, participación e igualdad

- Empleo, estable y de calidad.

- Territorialista, no se deslocaliza

- Desarrolla políticas sostenibles con el entorno en el que actúa

- Socialmente responsable

En torno a un catorce por ciento de la población mundial, forma parte, directamente, de una cooperativa, en cualquiera de sus modalidades (trabajo, agrarias, consumo, vivienda, crédito); es decir, más de ochocientos millones de personas están vinculadas al cooperativismo, en todo el mundo, inspirándose en los principios de la Alianza Cooperativa Internacional. $^{13}$

En Europa existen más de 70.000 cooperativas de trabajo, con cerca de un millón de personas socias que trabajan en ellas y, a su vez, generan empleos directos (trabajadores por cuenta ajena) que reúnen a más de un millón cien mil personas, vinculadas directamente a las cooperativas de trabajo; actuando en la práctica totalidad de los sectores económicos, entre los que sobresalen los sectores industrial y de servicios; dentro del sector servicios, tienen una incidencia destacable los servicios de atención a las personas: guarderías, residencias, centros de día/noche, ayuda a domicilio, centros educativos de 0 a 3 años.

En España, a fin del primer semestre del 2010, hay más de 17.700 cooperativas de trabajo asociado, con más de 270.000 empleos, generados de forma directa a través de socios

\footnotetext{
${ }^{13}$ La Alianza Cooperativa Internacional ACI agrupa a las cooperativas de todo el mundo. Está declarada como entidad consultiva de la ONU para el cooperativismo.
}

REVESCO No 105 - MONOGRÁFICO: La economía social y la igualdad de género -

ISSN: 1885-8031 - www.ucm.es/info/revesco 
y trabajadores por cuenta ajena, lo que supone casi el 1,5\% del empleo sobre el total de la población ocupada.

En los momentos actuales, la crisis económica ha provocado la destrucción de una gran parte del tejido empresarial y del empleo, de forma tal que ha retrocedido hasta los niveles, que existían hace más de cinco años. En este contexto, las cooperativas de trabajo han sabido perder menos empleos que otras figuras empresariales. En el segundo semestre de 2010, las cooperativas han experimentado una disminución del 2,6\% respecto del mismo periodo del año anterior.

Los estudios existentes y las cifras indicadas, tanto en el ámbito estatal como europeo, muestran que el cooperativismo de trabajo es un instrumento dinámico de creación de empleo y de progreso del espíritu empresarial, además de presentar grandes potencialidades como herramienta para el desarrollo de nuevos yacimientos de empleo, así como para la integración social y laboral de colectivos, como las mujeres, como se podrá comprobar.

\section{3.a) Sectores Productivos de actividad económica de las cooperativas de trabajo}

Los resultados de los Estudios, tanto en 2004 como en 2009, han demostrado que las actividades económicas de las cooperativas de trabajo en las que trabajan mujeres, contemplan la práctica totalidad de las actividades recogidas en el CNAE. Asimismo, se ha verificado que las actividades económicas que se perfilaban con una mayor presencia de mujeres, han seguido manteniendo esta tendencia e incluso incrementándola, así, por ejemplo, la subida en más de diez puntos en Otras actividades de servicios, la de más de cinco en Servicios sociales, sanitarios y de asistencia, o los incrementos en Educación, Hostelería y Transportes; hay que señalar la bajada más que significativa en Construcción (+ 6 puntos), Comercio (+ 5 puntos), Textil y confección o Metalurgia; en general, actividades relacionadas con la industria y aquéllas que durante el último año y medio han generado la mayor pérdida de empleos, como la construcción.

Agrupadas las actividades económicas en Sectores, se constata que el Sector Servicios ocupa a las tres cuartas partes de la población femenina de las cooperativas de trabajo.

REVESCO No 105 - MONOGRÁFICO: La economía social y la igualdad de género -

ISSN: 1885-8031 - www.ucm.es/info/revesco 


\section{3.b) Ámbito preferente de actuación de las cooperativas de trabajo}

Respecto del ámbito de actuación comercial -negocio- de las cooperativas de trabajo, se ha constatado que han aumentado en casi ocho puntos las que actúan en el ámbito estatal y se ha duplicado en el ámbito europeo/internacional, aún cuando la mayor parte de su actividad se ubica en el plano local/autonómico -casi un 77\%-, lo que ratifica el carácter territorialista de la cooperativa de trabajo.

\section{3.c) Facturación de las cooperativas de trabajo}

En el apartado de facturación relativa a los ejercicios 2007 y 2008, hay que señalar que el porcentaje de cooperativas de trabajo, con presencia de mujeres, que manifiestan encontrarse en el punto más alto de la variable facturación (Más de 6 millones de euros) permanece, prácticamente constante, en comparación con los datos obtenidos en el Estudio anterior.

Se destaca el incremento del porcentaje de cooperativas de trabajo, cuya facturación ha aumentado, en los tramos medios e inferiores, así, el mayor porcentaje de cooperativas se situaba en el tramo de 61.000 a 150.000 euros (años 2002-2003), en tanto que en las facturaciones de los ejercicios 2007 y 2008, el tramo mayor está referenciado a la cuantía de 151.000 a 300.000 euros. Esto sin lugar a dudas, supone un notable avance.

\section{3.d) Igualdad en las cooperativas de trabajo}

En el Estudio, se introdujo el análisis de la variable de la aplicación de la Igualdad en las cooperativas de trabajo, considerando que más allá del cumplimiento de la legislación vigente, estamos en presencia de un modelo empresarial, en el que el principio de la democracia participativa, no es sólo una referencia teórica, sino práctica, por lo que la Igualdad no constituye la excepción sino la normalidad en la actuación de la cooperativa.

REVESCO No 105 - MONOGRÁFICO: La economía social y la igualdad de género -

ISSN: 1885-8031 - www.ucm.es/info/revesco 
Analizando la Igualdad en la retribución, que como se ha señalado constituye uno de los déficits en España y en la UE, hallamos que, ante la pregunta de ¿Existe en la cooperativa una política de igualdad en las retribuciones entre hombres y mujeres? la respuesta de la gran mayoría ha sido afirmativa, en concreto el 86,9; ante un 13,1 que ha expresado una respuesta negativa. Y al preguntar sobre la existencia de una remuneración igual, para las mujeres y los hombres, por el mismo trabajo realizado, la respuesta ha sido contundente, ya que el $100 \%$ ha respondido afirmativamente.

\section{3.e) Personas que trabajan en las cooperativas de trabajo}

La edad media de las personas que forman las cooperativas de trabajo indica que se trata de personas que ya han rebasado el umbral que se considera de juventud, por cuanto se ubican a partir de los 25 años, con leves oscilaciones, a partir de esta edad, de un punto o punto y medio, hasta alcanzar la edad de la jubilación. ${ }^{14}$

\section{3.e) Vinculación de las mujeres con las cooperativas de trabajo}

Los vínculos de las personas que trabajan en las cooperativas de trabajo son diferentes en función de que las mismas sean socias o no de la cooperativa, así como que tengan contrato indefinido o no, en el supuesto de las personas asalariadas.

A este respecto, se ha comprobado que se mantiene la tendencia tanto del Estudio del 2004, como del Observatorio sobre la Formación Continua 2008, elaborado por Confederación Española de Cooperativas de Trabajo Asociado (COCETA) que indica que la relación de la mujer con la cooperativa de trabajo es, preferentemente, como Socia Trabajadora, con vinculación de carácter indefinido, de tal forma que un $86,60 \%$ de las mujeres de las cooperativas encuestadas, son socias con carácter indefinido, destacando un porcentaje de un $3,8 \%$ cuya vinculación, como socias, es de carácter temporal ${ }^{15}$.

\footnotetext{
${ }^{14}$ Conforme a los criterios establecidos en Geografía humana, al clasificar a las personas de acuerdo a su edad, obtenemos la cantidad de población joven (de 0 a 14 años), adulta (de 15 a 65 años) y anciana (de más de 65 años).

${ }^{15}$ Conviene citar que las legislaciones de cooperativas, permiten la existencia en las de trabajo asociado de socios de duración indefinida y temporal, cuya denominación es la de socios de duración determinada.
}

REVESCO No 105 - MONOGRÁFICO: La economía social y la igualdad de género -

ISSN: 1885-8031 - www.ucm.es/info/revesco 


\section{3.f) Perspectiva de género en los horarios de trabajo}

Dentro de este apartado, los resultados ofrecidos por el Estudio han reflejado que en un $90,9 \%$ de las personas encuestadas, no existen diferencias de horarios de trabajo, en función de la perspectiva de género. Esto significa que la cooperativa de trabajo tiene la misma flexibilidad /rigidez, tanto si se es hombre o mujer.

Analizadas, pormenorizadamente, las cooperativas de trabajo que han expresado la existencia de diferencias de jornada, entre mujeres y hombres, se ha obtenido como resultado, que la media de jornada semanal de los hombres en relación con la de las mujeres es 60 horas/45 horas. Igualmente, ante la evidencia de existencia de diferencias en las jornadas semanales entre las mujeres, según sean socias o no de la cooperativa, se ha comprobado que la jornada semanal de las mujeres socias respecto de las trabajadoras por cuenta ajena es de 60 horas/40 horas.

\section{3.g) Plan de Prevención de riesgos}

Conscientes de la necesidad, cada vez más creciente de que las empresas dispongan de una Plan de Prevención de riesgos, se ha analizado su existencia o no en las cooperativas de trabajo, habiéndose comprobado que el 92,2\% de las encuestadas manifiestan tener Plan de Prevención de riesgos; lo que implica que la existencia del citado Plan se ha convertido en una de las actuaciones habituales, dentro de las políticas empresariales de las cooperativas de trabajo. Dicho porcentaje puede considerarse bastante elevado, si se tiene en cuenta que en función de los datos del Observatorio estatal de las condiciones de trabajo, referidos al 2009, la media de empresas que tenían un Plan de Prevención, era del 64,7\%, si bien, en algunos sectores, como el Químico alcanzaba el 77,1\%. ${ }^{16}$

Porcentajes que sitúan a las cooperativas de trabajo en un alto nivel de responsabilidad para con las personas que trabajan en ellas. Es más, el Estudio también ha revelado que el $31,3 \%$ de las cooperativas de trabajo han incluido en el Plan de Prevención medidas

\footnotetext{
${ }^{16}$ Encuesta Nacional de Gestión de la Seguridad y la Salud en las empresas. 2009. Observatorio estatal de las condiciones de trabajo. MTIN. Instituto Nacional de Seguridad e Higiene. www.oect.es
}

REVESCO Nº 105 - MONOGRÁFICO: La economía social y la igualdad de género -

ISSN: 1885-8031 - www.ucm.es/info/revesco 
específicas para las mujeres. E incluso un $6,9 \%$ de las cooperativas de trabajo, tienen en el Plan de Prevención un protocolo en relación al acoso sexual.

Por último, en este apartado se ha analizado el grado de conocimiento de la existencia de la Ley para la igualdad efectiva de mujeres y hombres entre las cooperativas de trabajo, habiéndose obtenido como resultado que casi un $90 \%$ de las empresas encuestadas manifiestan conocerla y, de éstas, el 100\% han establecido medidas para la implantación de la Ley para la Igualdad.

Datos todos ellos, que vuelven a reiterar que la cooperativa de trabajo tiene como su valor más importante a las personas, sin distinción de género.

\section{PERFIL DE LAS MUJERES TRABAJADORAS EN LAS COOPERATIVAS DE TRABAJO}

\subsection{Perfil personal}

En el Estudio se consideraron diversas variables para elaborar el perfil personal de las mujeres que trabajan en las cooperativas de trabajo, obteniéndose como resultado que la media de edad de la mujer es de cuarenta años; respecto del estado civil, más del cincuenta por ciento está casada, aun cuando se ha producido una ligera disminución, respecto del Estudio del 2004; manteniéndose el resto de las categorías del estado civil, a excepción, de la situación de viviendo en pareja que ha aumentado, en este Estudio respecto del anterior, en más de un cincuenta por ciento.

En cuanto al número de hijos, la media por mujer trabajadora en las cooperativas de trabajo se sitúa en más de quince centésimas por debajo de la media de España, que a finales del 2008 era del 1,43. Esta tasa ha experimentado un ligero incremento, debido al mayor número de nacimientos entre la población inmigrante, debiendo señalarse que en las cooperativas de trabajo el número de mujeres inmigrantes aún es muy reducido, lo que de

REVESCO No 105 - MONOGRÁFICO: La economía social y la igualdad de género -

ISSN: 1885-8031 - www.ucm.es/info/revesco 
alguna manera, justificaría los índices de natalidad más bajos entre las mujeres de las cooperativas de trabajo.

En lo referente a la variable del nivel Formativo de las mujeres, se puede decir que el $55 \%$ ha manifestado haber finalizado estudios universitarios. En esta variable se ha producido un progreso significativo entre ambos Estudios (2004-2009), ya que ha disminuido el porcentaje de las mujeres sin estudios, aumentando el porcentaje en todos los demás niveles de formación, así en más de ocho puntos en el nivel de estudios universitarios, hasta alcanzar el $55 \%$ ya citado.

\section{3.- Perfil profesional}

Respecto del perfil profesional, el mayor porcentaje de mujeres en la cooperativa se ubica en la categoría del área de Administración, con un 32,1\%, frente a un 31,8\% del Estudio de 2004. Se ha reducido el porcentaje de mujeres trabajadoras no cualificadas, pasando del 4,1\% del Estudio del 2004, al actual 1,9\%; disminuye el de trabajadoras cualificadas, en dos puntos porcentuales, respecto del anterior estudio. Sí es de reseñar que el porcentaje de mujeres en puestos directivos ha disminuido en prácticamente dos puntos, del 18,8 del 2004 al 16,4\% del 2009, sin que las causas de esta minoración, hayan sido comprobadas. La categoría de mandos intermedios, sin embargo, ha experimentado un incremento de casi siete puntos.

El resultado del Estudio muestra que la mayoría de las mujeres que trabajan en las cooperativas de trabajo, lo hacen como socias trabajadoras indefinidas. Destacando el incremento en este grado de vinculación, de tal forma que del 70,9\% del Estudio del 2004, se ha pasado al $86,6 \%$. Igualmente es de reseñar la disminución de las mujeres contratadas temporalmente, ya que del 13,5\% del 2004 se ha pasado a un 3,8\%. Disminuye, asimismo, en casi un cincuenta por ciento las trabajadoras fijas, incrementándose, en casi dos puntos el porcentaje de socias trabajadoras temporales o de duración determinada, según la denominación que establezca la legislación sustantiva en cada comunidad autónoma. En definitiva la mujer en la cooperativa de trabajo presenta unos niveles de estabilidad laboral altos en lo que respecta a su vinculación con la entidad.

REVESCO No 105 - MONOGRÁFICO: La economía social y la igualdad de género -

ISSN: 1885-8031 - www.ucm.es/info/revesco 
Igualmente se ha querido conocer hasta qué punto incide en la conciliación personal/ laboral el número de hijos de las mujeres de las cooperativas de trabajo y el estado civil. A priori, podría parecer que el hecho de no tener cargas familiares, manifestado por el $72,1 \%$ implicaría una mayor conciliación personal/laboral. Sin embargo, en función de los resultados obtenidos, se puede decir que las mujeres de las cooperativas de trabajo, concilian personal y laboralmente, en la misma intensidad, con independencia de su estado civil y del número de hijos; produciéndose la variable más alta de equiparación de conciliación e hijos en el estado civil de viuda, siendo la media más alta de conciliación la correspondiente al estado civil de divorciada.

En cuanto al grado de conciliación, el Estudio refleja que, como regla general, la gran mayoría de las mujeres que trabajan en las cooperativas sienten conciliada su vida laboral y familiar, habiéndole otorgado la calificación de elevada. A partir de aquí, si se analiza por categorías laborales, se puede comprobar que las mujeres que menos concilian son las trabajadoras no cualificadas, en tanto que el mayor porcentaje de conciliación se produce entre las mujeres que realizan tareas de administración, casi el $90 \%$. Igualmente, las mujeres que ocupan puestos directivos, manifiestan en más de un $40 \%$ de los casos, tener un elevado nivel de conciliación y, un porcentaje inferior al 20\%, se declara conciliadas en grado absoluto. Puede que este elevado porcentaje de conciliación en las cooperativas de trabajo, venga dado por la existencia de ayudas para las mujeres, casi el $80 \%$ de las mujeres encuestadas han indicado que tienen ayudas de carácter personal para conciliar la vida personal y la laboral. Ayuda que normalmente se concentra en familiares, existiendo asimismo, un buen número de redes sociales, que posibilitan que las mujeres puedan conciliar. Si comparamos los datos con los del Estudio de 2004, los porcentajes en las diferentes clases de ayudas han experimentado un crecimiento significativo. Así, mientras que en Ayudas personales, tan sólo las declaraban un 30,9\% de las mujeres, ahora, ha subido hasta el $80 \%$. Respecto de las Ayudas laborales se sitúan en torno al $17,4 \%$, lo que supone un incremento de más de diez puntos respecto del Estudio de 2004. Por último, las Ayudas institucionales son las únicas que han disminuido, en 1,4 puntos, si bien es cierto que era un porcentaje reducido en el 2004, en este Estudio aún lo ha sido más.

REVESCO No 105 - MONOGRÁFICO: La economía social y la igualdad de género -

ISSN: 1885-8031 - www.ucm.es/info/revesco 
Otra de las variables que facilita la conciliación laboral y familiar es el entorno en el que se trabaja, en este caso, la cooperativa de trabajo. El Estudio revela que cuando se ha preguntado si se consideraba que trabajar en una cooperativa de trabajo, como modelo empresarial avanzado y participativo, facilitaba la conciliación personal y laboral, una amplia mayoría, casi la mitad de las mujeres, han indicado que efectivamente sí facilita la conciliación; considerando, más de una cuarta parte de las mujeres encuestadas que "es mucha la influencia de la cooperativa como fórmula empresarial para facilitar la conciliación”.

\section{CIFRAS PARA UNA REALIDAD SOCIAL Y LABORAL DE LAS MUJERES EN LAS COOPERATIVAS DE TRABAJO ASOCIADO EN ESPAÑA}

- De cada cinco personas que hay en una cooperativa, tres son mujeres.

- Los sectores: Servicios $(75,80 \%)$ e Industria (21,20\%) concentran el empleo femenino en las cooperativas de trabajo.

- Consultoría empresarial $(11,10 \%)$ y educación $(11,10 \%)$, servicios a las personas $(16,20 \%)$ y otras actividades de servicios $(24,20 \%)$ concentran la actividad de las mujeres.

- Respecto de la edad de las mujeres se sitúa en una media de 39,27 años.

- Más de la mitad de las mujeres que trabajan en las cooperativas de trabajo tienen estudios universitarios.

- El estado civil mayoritario es el de casada (55,6\%), seguido del de soltera (22,0\%).

- La media de hijos por mujer en las cooperativas de trabajo es de 1,09.

- En los puestos directivos se alcanza una media del 16,4\%

- Respecto de la relación de la mujer con la cooperativa se concentra en socias trabajadoras indefinidas $(86,60 \%)$.

- El $90,90 \%$ de las mujeres manifiesta que no existen diferencias entre las horas trabajadas por hombres y mujeres.

- El porcentaje de mujeres responsables de cooperativas alcanza el 39,7\%.

- La conciliación laboral y familiar sigue siendo una constante en las cooperativas de trabajo, más de un 56,4\%, declara sentirse bastante conciliada.

REVESCO No 105 - MONOGRÁFICO: La economía social y la igualdad de género -

ISSN: 1885-8031 - www.ucm.es/info/revesco 
- Más de la mitad de las mujeres que trabajan en las cooperativas considera que el modelo cooperativo es idóneo para la conciliación laboral y familiar.

- El 87,9\% manifiesta la existencia de políticas de acceso de la mujer a puestos de dirección.

- El 86,9\% de las mujeres indica que en la cooperativa existen políticas de igualdad en la retribución entre hombres y mujeres.

- El $100 \%$ de las mujeres manifiestan que existe igualdad de remuneración por el mismo trabajo entre hombres y mujeres

- El 92,2\% de las cooperativas tienen un Plan de Prevención de Riesgos.

- El 31,3\% de las cooperativas que tienen un Plan de Prevención de Riesgos, incluyen medidas específicas para mujeres.

- El 6,9\% de las cooperativas tienen un protocolo de actuación establecido en relación al acoso.

\section{CONCLUSIONES}

En función de la información recabada en el Estudio, se puede decir, con carácter general, que la situación de la mujer en España, respecto de las diferentes variables que se han analizado se encuentra en un nivel inferior al que puede tener en otros países de la Unión Europea.

Al mismo tiempo se ha constatado que en las variables analizadas, en el marco de la Unión Europea se producen desigualdades entre mujeres y hombres, así, en lo que respecta a los aspectos laborales, hay que citarlas en las remuneraciones, las jornadas, en la distribución de la clave "tiempo", lo que lleva a reflexionar sobre la necesidad de promover medidas legislativas, tendentes a la eliminación de estas desigualdades, así como medidas formativas basadas en el principio de igualdad entre mujeres y hombres, que propicien, a través de la formación, un cambio en la mentalidad de la ciudadanía.

Los avances experimentados en los últimos cinco años, que han llevado a un cierto avance, no significa que no existan desigualdades y diferencias, en temas tan concretos como

REVESCO No 105 - MONOGRÁFICO: La economía social y la igualdad de género -

ISSN: 1885-8031 - www.ucm.es/info/revesco 
el acceso al empleo, la retribución o el uso del tiempo. Todo ello lleva a reivindicar la necesidad, en España, de avanzar más rápidamente en la puesta en marcha de las políticas precisas para que la ejecución de la Ley para la igualdad, prosiga sin retrasos; considerando que no se puede dar opción a que la actual situación económica obstaculice su progreso.

Analizada la situación de la mujer en las cooperativas de trabajo y comparada con las variables existentes en España, se puede concluir que, con carácter general, es mejor.

Indicadores tan significativos como la retribución, las jornadas de trabajo, revelan que las mujeres y los hombres son iguales en las cooperativas de trabajo.

Hay que destacar el grado de concienciación en las cooperativas, en relación a las políticas de prevención de riesgos con respecto a otras figuras empresariales. Esta labor preventiva se destaca también en la existencia de medidas concretas para las mujeres que trabajan en las cooperativas.

Se ha comprobado cómo la cooperativa de trabajo facilita la formación tanto a las mujeres como a los hombres, siendo, sin embargo, destacable el alto porcentaje de mujeres que participan en las acciones formativas, superando al de los hombres.

La mujer se vincula a la cooperativa de trabajo, de forma mayoritaria a través del estatus de socia trabajadora; en este sentido, las medidas llevadas a efecto desde el Estudio del 2004, entre las que destacan la política remunerativa, las jornadas flexibles, acciones de empoderamiento, han posibilitado el aumento de mujeres socias, respecto de las trabajadoras por cuenta ajena, disminuyendo el volumen de temporalidad de las mujeres, lo que otorga una estabilidad a la empresa cooperativa que le confiere ventaja competitiva. El nivel de experiencia, adquirido por una práctica continua y estable, en una tarea u oficio genera una mayor calidad en el producto o servicio que se realiza.

La existencia de políticas de conciliación de la vida personal y familiar con la laboral, es una de las ventajas competitivas de las cooperativas de trabajo. Las personas que trabajan motivadas, realizan mejor su trabajo y son más productivas.

En definitiva, se constata que la cooperativa de trabajo, por los valores que incorpora como la solidaridad, la igualdad, el predominio de la persona sobre el capital, el entronque

REVESCO No 105 - MONOGRÁFICO: La economía social y la igualdad de género -

ISSN: 1885-8031 - www.ucm.es/info/revesco 
con el territorio, unidos a un forma de gestión empresarial cuyo objetivo principal es el mantenimiento del empleo de las personas que la forman, hacen de la cooperativa de trabajo el modelo empresarial idóneo para las mujeres.

\section{BIBLIOGRAFÍA}

ADECCO. FUNDACIÓN ADECCO. IV Informe Perfil de la Mujer Trabajadora. Madrid 2010. Disponible en Internet:

www.fundacionadecco.es/_data/SalaPrensa/SalaPrensa/Pdf/163.pdf

AGRUPACIÓN DE SOCIEDADES ASTURIANAS DE TRABAJO ASOCIADO. ASATA. Aplicación de la ley de igualdad en las empresas de Economía Social de Gijón. Gijón. 2009

ALIANZA COOPERATIVA INTERNACIONAL. ACI. Principios cooperativos. Disponible en Internet: http://www.ica.coop/coop/principles.html

COMISIÓN EUROPEA. Europa 2020. Una estrategia para un crecimiento inteligente, sostenible e integrador. Comunicación de la Comisión.. Bruselas, 3.3.2010. COM (2010) 2020. Disponible en Internet: ec.europa.eu/commission_20102014/president/.../20100303_1_es.pdf

CONFEDERACIÓN ESPAÑOLA DE COOPERATIVAS DE TRABAJO ASOCIADO. COCETA. Estudio sobre la realidad social y laboral de las mujeres en el Cooperativismo de trabajo asociado en España. Madrid. 2004.

CONFEDERACIÓN ESPAÑOLA DE COOPERATIVAS DE TRABAJO ASOCIADO. COCETA. Observatorio Permanente de Formación Continua en Cooperativas de Trabajo Asociado. .Madrid. 2008.

ECOTEC Research \& Consulting Ltd. ECORYS España. NIPO: 803-09-001-9. Ref: 828P.E.2007. Ministerio de Igualdad. Secretaría General de Políticas de Igualdad. Instituto de la Mujer. Estudios e Investigaciones. Evaluación de Políticas de Igualdad en el ámbito laboral en España. Año 2007-.Año 2008.. Disponible en Internet: http://www.inmujer.migualdad.es/mujer/mujeres/estud_inves/82...

REVESCO No 105 - MONOGRÁFICO: La economía social y la igualdad de género ISSN: 1885-8031 - www.ucm.es/info/revesco 
EUROSTAT EUROPEAN COMMISION. Reconciliation between work, private and family life in the European Union. Eurostat Statistical books. 2009. Disponible en Internet: http://epp.eurostat.ec.europa.eu/cache/ITY_OFFPUB/KS-78-09-9

FUNDACIÓN TRIPARTITA PARA LA FORMACIÓN Y EL EMPLEO. Formación continua. Informe de los Resultados 2006. Principales indicadores. Participación de las mujeres, en la actividad formativa. Página 78. http://www.fundaciontripartita.org/almacenV/publicaciones/documentos/45735_7878200 8145632.pdf

FUNDACIÓN TRIPARTITA PARA LA FORMACIÓN Y EL EMPLEO. Estadísticas participantes formación continua 2006. Disponible en Internet: http://www.fundaciontripartita.org/index.asp?MP=6\&MS=24\&MN=2

LEY 31/1995, de Prevención de Riesgos Laborales, de 8 de noviembre. BOE. Número 269.10 de noviembre 1995 , pp. 32590-32611

LEY 27/1999, de Cooperativas, de 16 de julio. BOE. Número 170. 17 de julio de 1999, pp. 27027-27062

LEY 39/1999, para promover la conciliación de la vida familiar y laboral de las personas trabajadoras, de 5 de noviembre. BOE. Número 266. 6 de noviembre 1999, pp.3893438941

LEY ORGÁNICA 3/2007, de 22 de marzo, para la igualdad efectiva de mujeres y hombres.BOE. Número 71.23 de marzo de 2007, pp. 12611-12645

MINISTERIO DE TRABAJO E INMIGRACIÓN. Evaluación de políticas de igualdad en el ámbito laboral en España (año 2007-2008).

www.inmujer.migualdad.es/MUJER/mujeres/estud_inves/828.pdf

MINISTERIO DE TRABAJO E INMIGRACIÓN. Instituto Nacional de Seguridad e Higiene en el Trabajo. Encuesta Nacional de Gestión de la Seguridad y Salud de las Empresas. ENGE. 2009. Disponible en Internet:

http://www.oect.es/portal/site/Observatorio/menuitem.542e5ada348272e409b11276b5d06

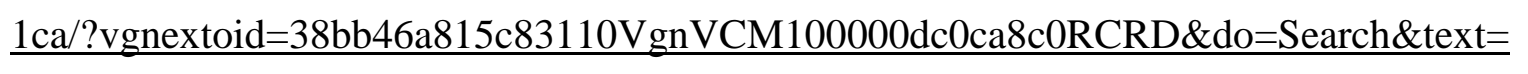
encuesta+nacional+seguridad+empresas+

MINISTERIO DE TRABAJO E INMIGRACIÓN. Datos estadísticos. Disponible en Internet:http://www.mtin.es/es/sec_trabajo/autonomos/economiasoc/EconomiaSocial/estadisticas/

REVESCO No 105 - MONOGRÁFICO: La economía social y la igualdad de género -

ISSN: 1885-8031 - www.ucm.es/info/revesco 
OECD. Publications. Education at a Glance. 2008. Paris 2008. Informe de la Organización para la Cooperación y el Desarrollo Económico (OCDE), Panorama de la educación 2008. Ministerio de Educación y Ciencia.2008

VARA MIRANDA, M.J. Funciones de la economía social en el modelo de desarrollo económico. I.C.E. mayo, número 729. Pp. 9-17. .(1994)

REVESCO No 105 - MONOGRÁFICO: La economía social y la igualdad de género ISSN: 1885-8031 - www.ucm.es/info/revesco 